ESAIM: PROCEEDINGS AND SURVEYS, December 2016, Vol. 55, p. 23-40

Emmanuel FRÉNOD, Emmanuel MAITRE, Antoine ROUSSEAU, Stéphanie SALMON and Marcela SZOPOS Editors

\title{
HYDROMORPHO: A COUPLED MODEL FOR UNSTEADY STOKES/EXNER EQUATIONS AND NUMERICAL RESULTS WITH FEEL++ LIBRARY*
}

\author{
Nora AÏssiouene ${ }^{1}$, Tarik Amtout ${ }^{2}$, Matthieu Brachet ${ }^{3}$, Emmanuel Frenod $^{4}$, \\ Romain Hild ${ }^{5}$, Christophe Prud'homme ${ }^{6}$, Antoine Rousseau ${ }^{7}$ and Stephanie \\ SALMON $^{8}$
}

\begin{abstract}
We propose to couple the Exner equation with the Stokes equations to model the bedload sediment in geophysical flows. This work is a preliminary study to directly model the hydrodynamic flow by the unsteady Stokes equation instead of the classical shallow water equation. We focus in this proceeding on the coupling applying fluid structure interaction approach to morphodynamical behavior. In other words, we follow the approach of fluid interaction models replacing the structure equation by the Exner equation. The aim of this work is to validate the proposed procedure. These equations are solved by finite element method using the library FEEL++.
\end{abstract}

Résumé. Nous proposons de coupler l'équation d'Exner avec les équations de Stokes afin de modéliser le transport des sédiments par charriage. Ce travail est une étude préliminaire pour modéliser le flux hydrodynamique par les équations instationnaires de Stokes à la place du choix classique des équations de Saint-Venant. Ici, nous nous concentrons sur l'utilisation d'une approche interaction fluide structure pour le couplage, c'est-à-dire remplacer l'équation de structure par l'équation d'Exner. Le but de ce travail est de valider la procédure proposée. Ces équations sont résolues par la méthode des élements finis en utilisant la bibliothèque FEEL++.

\section{INTRODUCTION}

Many hydrodynamic studies have been done to understand and predict the dynamics of sediments at the bottom of flows which is a significant and complex process for many geophysical situations. Morphodynamics modelling is a broad subject whose principles can be found in several references [34], [35]. We can distinguish two types of sediment transport, the suspended load and the bedload. In this proceeding, we focus on the bedload transport and its impact on the hydrodynamics. The difficulty remains in the necessity to couple the

* We thank the GDR EGRIN for its financial support and the CIRM for its warm welcome during the CEMRACS.

${ }^{1}$ Inria - CEREMA - Sorbonne Universités, UPMC Univ Paris 06 - CNRS, UMR 7598, Laboratoire Jacques-Louis Lions

2 Faculté des Sciences et Techniques Tanger, Maroc

3 Institut Elie Cartan de Lorraine, Université de Lorraine, Site de Metz, Bât. A Ile du Saulcy, F-57045 Metz Cedex 1

${ }^{4}$ Université Bretagne-Sud, LMBA UMR 6205, Vannes

${ }^{5}$ Institut de Recherche Mathématique Avancée, Strasbourg

${ }^{6}$ Institut de Recherche Mathématique Avancée, Strasbourg

7 Inria and Institut Montpelliérain Alexander Grothendieck, Team LEMON, Bat 5 - CC05 017, 860 rue Saint-Priest, 34095 Montpellier Cedex 5, France

${ }^{8}$ Laboratoire de Mathématiques de Reims, Fédération ARC CNRS 3399, Université de Reims Champagne-Ardenne

(C) EDP Sciences, SMAI 2017 
sediment transport models with the hydrodynamic models, and then to develop a robust and stable numerical method.

On the one hand, the sediment transport is usually modeled by the classical Exner equation [37] and several laws of transport have been proposed (see [21] to have details on some classical laws) by physical arguments or closure relations. On the other hand, models as shallow water equations are used to model the hydrodynamics, and recently in $[12,21]$ a model derived from the Navier-Stokes equations that has an energy balance.

Concerning the numerical methods that have been established for these models, the main numerical schemes are developed for the hyperbolic systems with source terms for the hydrodynamic flow (see [9], [25]). Therefore, finite volume schemes are applied for the shallow water system $[1,2,23,38]$. The problem lies in the coupling of the numerical schemes. Indeed, in the shallow water models, the topography is a source term and the Exner equation gives the evolution of the bottom in terms of the fluid velocity. Then, two strategies are distinguished, the splitting one and the non-splitting one (see [4]). The splitting methods are easier to implement but generate instabilities in specific situations (see [14]). On the contrary, more complicated models, for instance involving relaxation, have to be used to take into consideration the fully coupled model [3,29].

Notice that the shallow water model is based on a hydrostatic assumption. It is deduced from the Navier Stokes equations, neglecting the vertical acceleration (see [23]). Many other free surface models have been developed to take into account non-hydrostatic effects with vertically averaged models: see $[8,10,11,18,32]$. Contrariwise, for this study we choose to conserve the $z$ coordinate in our model, which raises the question of time-depending domain when the bathymetry changes with time: this coupling between fluid motion (including vertical effects) and domain evolution is at the core of this paper.

This objective being stated, we start with the simplest possible model, a 2D $(x-z)$ Stokes equation. We couple this equation for the fluid with the Exner model since our computational domain moves as times goes by. We choose to use the Grass law for the bedload formula (see Equation (10)) which is one possible law among others. As for the time coupling between hydrodynamical and morphological processes, we choose to use a monolithic scheme rather than a splitting method: such refinements (that can prove to be very important, see [14]) are beyond the scope of our work.

Let us now focus on the main feature of this work: the use of fluid-structure interaction techniques (see $[13,26]$ ) for the coupling between Stokes and Exner equations. From the numerical viewpoint, we decided to use finite elements and the open software Feel++ [39-41] that are well adapted to fluid-structure interaction (ALE implementation, see [26]) and parallelization for large 3D computations.

The article is organized as follows, the first part is devoted to the description of the fluid model and the sediment model at the bottom. In a second part, it is explained why a method like the ALE is necessary to couple the models. The third part establishes a complete ALE formulation of the Stokes-Exner model. Then, a variational formulation is given with the different boundary conditions that we explore. Finally, some numerical results are presented to evaluate the model and the method used to solve the problem.

\section{THE MODEL}

In this part, we introduce various equations for our coupled system. Section 1.1 is devoted to the unsteady Stokes equations (dimension 2, $x-z$ ) that we supplement with appropriate boundary conditions. Section 1.2 is dedicated to the bottom boundary condition, located at the (moving) boundary where the fluid model is coupled with the Exner equation for bedload. Before recalling the complete coupled system in section 1.4, we present in Section 1.3 the ALE implementation of our model.

We start with a model domain $\Omega(t)$ and a specific boundary to represent the topography. We consider the domain as a moving domain depending on the bottom. Let us introduce the domain with the following 
definitions:

$$
\Omega(t)=\left\{(x, z) \in \mathbb{R}^{2} \quad \mid \quad 0 \leq x \leq l, \quad b_{z}(x, t) \leq z \leq 1\right\}
$$

where $l>0$ is the cavity length and $b_{z}(x, t)$ is the bottom topography in $x$ at time $t$. We also denote by $\Gamma(t)=\Gamma_{\text {in }}(t) \cup \Gamma_{\text {out }}(t) \cup \Gamma_{s} \cup \Gamma_{b}(t)$ the boundaries (see Figure 1):

- $\Gamma_{\text {in }}(t)=\{0\} \times\left[b_{z}(0, t), 1\right]$

- $\Gamma_{\text {out }}(t)=\{l\} \times\left[b_{z}(l, t), 1\right]$,

- $\Gamma_{s}=[0, l] \times\{1\}$,

- $\Gamma_{b}(t)=\left\{(x, z) \in \mathbb{R}^{2}\right.$ s.t. $\left.z=b_{z}(x, t), x \in[0, l]\right\}$.

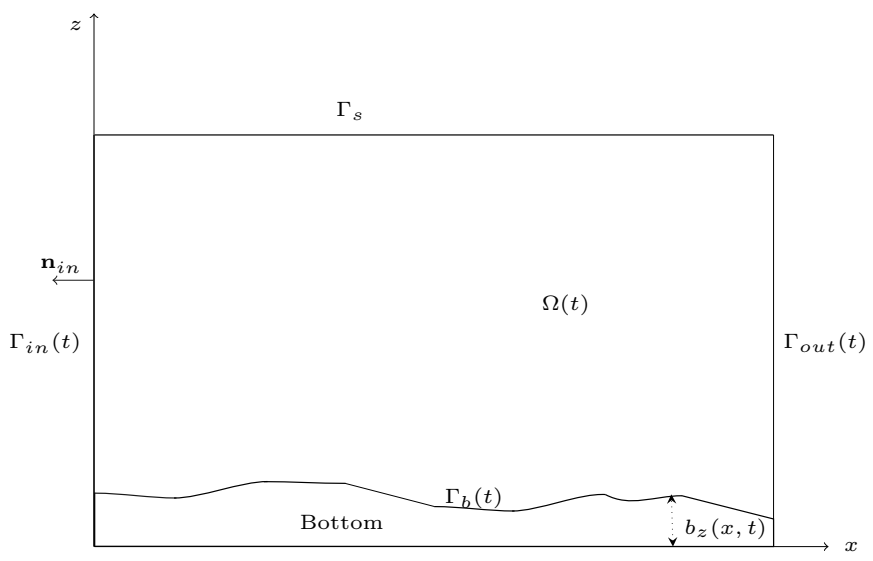

FiguRE 1. Definition of the domain

The coupled model leads to solving the non-steady Stokes problem in the fluid domain $\Omega(t)$ and the Exner equation to give the boundary $\Gamma_{b}(t)$. The issue is to model the fluid process in interaction with the sediment transport at the bottom. In the following sections, we describe the equations chosen for the fluid in the domain $\Omega(t)$ with usual boundary conditions for the boundary $\Gamma$. Then we propose to use Exner equation to make the boundary $\Gamma_{b}$ move.

\subsection{Hydrodynamical Model}

We consider the unsteady Stokes problem on the domain $\Omega(t)$

$$
\begin{aligned}
\rho \frac{\partial \mathbf{u}}{\partial t}-\mu \triangle \mathbf{u}+\nabla p & =0 \text { on } \Omega(t), \\
\operatorname{div}(\mathbf{u}) & =0 \text { on } \Omega(t),
\end{aligned}
$$

where $\mathbf{u}=(u, w)^{T}$ is the velocity of the fluid, $p$ is the pressure, $\mu>0$ is the dynamic viscosity and $\rho$ is the density. From now on, we will use $\rho=1$. This problem is completed by the boundary conditions detailed hereafter.

A crucial issue is to have judicious boundary conditions at the interface between the fluid and the topography. The physical behavior of the sediment transport studied here implies an impermeability boundary condition, then a constraint on the normal component of the velocity has to be done. Concerning the other boundaries, we will consider a model test case on which we simulate a flow on the pseudo free surface $\left(\Gamma_{s}\right.$ in our case). Then, 
we impose the velocity on the surface boundary $\Gamma_{s}$ by a Dirichlet condition and free boundary conditions, using Neumann boundary conditions for the velocity, at the inlet and outlet

$$
\begin{aligned}
\mathbf{u} & =\mathbf{g}_{1} \text { on } \Gamma_{s} \\
\boldsymbol{\sigma} \mathbf{n}=\mu \frac{\partial \mathbf{u}}{\partial \mathbf{n}}-p \mathbf{n} & =\mathbf{g}_{2} \text { on } \Gamma_{\text {in }}(t) \cup \Gamma_{\text {out }}(t), \\
\mathbf{u} \cdot \mathbf{n} & =0 \quad \text { on } \Gamma_{b}(t) \\
\boldsymbol{\sigma n} \cdot \boldsymbol{\tau}=\mu \frac{\partial \mathbf{u}}{\partial \mathbf{n}} \cdot \boldsymbol{\tau} & =g_{3} \text { on } \Gamma_{b}(t)
\end{aligned}
$$

where $\boldsymbol{\sigma}$ is the stress tensor defined by:

$$
\boldsymbol{\sigma}=(\mu \nabla \mathbf{u}-p \mathbb{I} d)
$$

The condition (4) imposes the force by using Dirichlet condition.

The condition (5) lets free the velocity at the inlet and at the outlet.

The condition (6) imposes the normal component of the velocity to be null at the bottom. It is the condition of impermeability of the domain.

The condition (7) lets free the tangential component of the velocity at the bottom. It is needed to have a displacement of the bottom.

\subsection{Morphodynamics model}

The sediment dynamics is based on the formulation of a sediment continuity equation stating that the time variation of the sediment layer in a certain volume is due to the net variation of the solid transport through the boundaries of the volume. The mathematical expression of such law is known as the Exner equation [33] presented in this form:

$$
\frac{\partial b_{z}}{\partial t}+\xi \frac{\partial Q}{\partial x}=0 \quad \forall x \in[0, l], \forall t \in[0, T]
$$

where $b_{z}(x, t)$ is the bed elevation, $\xi$ is defined by $(1-p)^{-1}$ where $p$ is the material porosity and $\mathrm{Q}$ denotes the solid transport discharge along the $\mathrm{x}$ coordinate influenced by the velocity $\mathbf{u}$. The formulation of the bedload discharge Q can be based on deterministic laws ( [5], [19], [42]) or in probabilistic methods ( [20], [30]), often supported by experimentation. Grass [27] discussed one of the most basic sediment transport laws that can be written in one dimension as:

$$
Q=a|\mathbf{u}|^{3 / 2}
$$

where $0<a<1$ is an empirical parameter depending of the type of the sediments, it takes into account the effects due to the grain size and the kinematic viscosity. For the problem studied in this work, the velocity taken into consideration in the Grass formula is reduced to the tangential part $\mathbf{u}_{\tau}$ since we impose an impermeability condition on the interface, see the boundary condition (6).

For the sake of clarity, we will consider the Exner equation under the form:

$$
\frac{\partial b_{z}}{\partial t}+\frac{\partial Q}{\partial x}=0 \quad \forall x \in[0, l], \forall t \in[0, T]
$$

where $Q=\alpha\left|\mathbf{u}_{\tau}\right|^{3 / 2}$ and $\alpha=\xi a$. 


\subsection{Arbitrary Lagrangian Eulerian (ALE) Method}

We now want to couple the two models previously described. The issue is to solve the unsteady Stokes equations with a moving boundary $\Gamma_{b}$. In fluid mechanics, one can enumerate two ways to represent a problem: Lagrangian and Eulerian formulation. On the one hand, Lagrangian formulation is similar to keep track of the location of each fluid particles. The velocity $\mathbf{u}$ and the density $\rho$ depend only on $\mathbf{x}_{\mathbf{0}}$ the initial position of the particles and on $t$ the time. Then, the time derivative of a quantity $F$ is given by the total time derivative :

$$
\frac{D F}{D t}
$$

On the other hand, the main idea of the Eulerian method is to fix a system of coordinates and follow the flux of particles. In this case, the velocity $\mathbf{u}$ and the density $\rho$ depend on $\mathbf{x}$ the position in a global system of coordinates and $t$ the time. Now, for a function $F$, the time derivative is given by :

$$
\frac{\partial F}{\partial t}+\mathbf{u} \cdot \nabla F
$$

The relation between the Eulerian and the Lagrangian time derivatives is:

$$
\frac{D}{D t}=\frac{\partial}{\partial t}+\mathbf{u} \cdot \nabla
$$

Then, the idea of the ALE method is to combine the Eulerian and the Lagrangian method in order to take into consideration the boundary displacement at each iteration, which represents the bottom in our case. The goal is to avoid remeshing the domain at each time iteration. This method was first developped for finite difference in $[22,36]$ and scope to finite element methods in $[16,17]$ and [6]. In 2004, [13] built a method for great order elements. This has been widely used in Fluid Structure Interaction (FSI) on which it is usual to have a fluid equation like unsteady Stokes or Navier Stokes in the fluid domain and an elasticity equation for the structure. This is used in the simulation of blood flow in arteries for instance (see [13], [26]). In the context of the sediment transport, the bottom plays the role of the structure in the classical methods. Then the idea is to use the analogy of these methods for the coupled Stokes Exner model.

As the goal is to avoid remeshing the domain, the clue is to use a Lagrangian description to describe the bottom displacement and a Eulerian description for the fluid model. First, we define a reference domain on which the topography is described by a Lagrangian description. Secondly, we consider the physical domain $\Omega(t)$ on which the equations of the fluid evolves. Then, it is necessary to define an application able to make the link between the two domains. In the following, we write $\widehat{\cdot}$ all quantities concerning the reference domain. For the sake of clarity, we choose $\widehat{\Omega}$ the rectangular domain $[0, l] \times[0,1]$ and the following boundaries :

- $\widehat{\Gamma}_{i n}=\{0\} \times[0,1]$,

- $\widehat{\Gamma}_{s}=[0, l] \times\{1\}$,

- $\widehat{\Gamma}_{\text {out }}=\{l\} \times[0,1]$,

- $\widehat{\Gamma}_{b}=[0, l] \times\{0\}=\widehat{\gamma}_{b} \times\{0\}$ where $\widehat{\gamma}_{b}=[0, l]$.

The relation between the reference domain $\widehat{\Omega}$ and the physical domain $\Omega(t)$, is made by an ALE map (see figure 2 ), defined by :

$$
\mathcal{A}^{t}:\left\{\begin{array}{lll}
\widehat{\Omega} & \longrightarrow & \Omega(t) \\
\widehat{\mathbf{x}} & \longmapsto & \mathbf{x}(\widehat{\mathbf{x}}, t)
\end{array} .\right.
$$




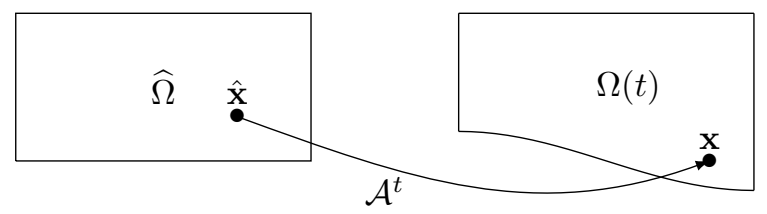

Figure 2. The ALE map

Therefore the point $\mathbf{x}(t) \in \Omega(t)$ is obtained by:

$$
\mathbf{x}(t)=\mathcal{A}^{t}(\widehat{\mathbf{x}})=\widehat{\mathbf{x}}+\widehat{\mathbf{d}}_{\delta}(\widehat{\mathbf{x}}, t),
$$

where $\widehat{\mathbf{d}}_{\delta}(\widehat{\mathbf{x}}, t)$ is the displacement of $\widehat{\mathbf{x}}$ between $\widehat{\Omega}$ and $\Omega(t)$. Notice that $\mathbf{x}(t)$ is time dependent. Then, we can define the velocity of the mesh:

$$
\widehat{\mathbf{w}}(\widehat{\mathbf{x}}, t)=\frac{\partial \mathcal{A}^{t}}{\partial t}(\widehat{\mathbf{x}})=\frac{\partial \widehat{\mathbf{d}}_{\delta}}{\partial t}(\mathbf{x}, t)
$$

where $\widehat{\mathbf{w}}(\hat{\mathbf{x}}, t) \in \mathbb{R}^{d}$ is defined for $\hat{\mathbf{x}} \in \widehat{\Omega} \times \mathbb{R}^{+}$. To take into consideration the velocity of the mesh into the fluid equation, it is necessary to define it in the fluid domain $\Omega(t)$, namely:

$$
\begin{aligned}
& \mathbf{w}:(\mathbf{x}, t) \in \Omega(t) \times \mathbb{R}^{+} \rightarrow \mathbb{R}^{d}, \\
& \mathbf{w}=\widehat{\mathbf{w}} \circ\left(\mathcal{A}^{t}\right)^{-1} .
\end{aligned}
$$

This definition will allow us to rewrite the fluid equation with an Eulerian description, taking into account the displacement of the mesh. The last step of the method leads to determine the equation of the displacement $\widehat{\mathbf{d}}_{\delta}$ in $\widehat{\Omega}$. In practice, $\mathbf{d}_{\delta}$ is the solution of a PDE like harmonic or Wislow equation. For the sake of simplicity, we will work with harmonic extension that allows to have a smooth mesh. We often need to transport an equation from $\widehat{\Omega}$ to $\Omega(t)$ and mutually. Let $u: \Omega(t) \times \mathbb{R}^{+} \longrightarrow \mathbb{R}^{d}$, then the corresponding map in $\widehat{\Omega}$ is $\widehat{u}=u \circ \mathcal{A}^{t}$. If $\mathcal{D} \mathbf{u} / \mathcal{D} t$ is the time-derivative of $u$ in ALE, we have the following equation:

$$
\frac{\mathcal{D} \mathbf{u}}{\mathcal{D} t}=\left.\frac{\partial \mathbf{u}}{\partial t}\right|_{x}+\mathbf{w} \cdot \nabla \mathbf{u}
$$

- Notice that if $\mathbf{w}=\mathbf{u}$, the mesh is moving with the particles so the description is Lagrangian.

- If $\mathbf{w}=\mathbf{0}$, the mesh does not move and the description is Eulerian.

\subsection{Coupled model}

In this part, we focus on the coupled model. We denote by $\widehat{\mathbf{x}}$ a point in the reference domain $\widehat{\Omega}$ and by $\Omega(t)$ the deformed domain after the transformation. The deformation of the mesh leads to consider the derivative $\mathcal{D}$ defined by (18) which is also called the ALE derivative where the velocity $\mathbf{w}$ is defined as: $\widehat{\mathbf{w}}=\left.\frac{\partial \widehat{\mathbf{d}}_{\delta}}{\partial t}\right|_{\hat{x}}$ and represents the velocity of the displacement of the mesh, that is to say the velocity of the particle in the referential domain. This allows to write the fluid model with a moving mesh on $\Omega(t)$. Concerning the boundary conditions, we still consider a slip boundary condition at the interface between the topography and the fluid. The complete model is composed of the equation of the fluid in two dimensional domain, the equation of the topography in one dimensional domain and the ALE equation in two dimensional domain. According to (18), the coupled model is written as follows : 


\subsubsection{Fluid equation}

The fluid equation is given by :

$$
\begin{aligned}
\frac{\mathcal{D} \mathbf{u}}{\mathcal{D} t}-(\mathbf{w} \cdot \nabla) \mathbf{u}-\mu \triangle \mathbf{u}+\nabla p=\mathbf{F} & \text { on } \Omega(t), \\
\operatorname{div}(\mathbf{u})=0 & \text { on } \Omega(t), \\
+\mathbf{B C}, &
\end{aligned}
$$

where the boundary conditions are those of section 1.1. In particular, condition (7) depends on the bottom topography.

\subsubsection{Bottom equation}

We consider the one dimensional domain $\widehat{\gamma}_{b}=[0, l]$, on which the bottom topography at position $\widehat{x} \in \widehat{\gamma_{b}}$ and time $t>0$ is defined by the Exner equation (11):

$$
\begin{aligned}
\frac{\partial \hat{b}_{z}(\hat{x}, t)}{\partial t}+\frac{\partial \hat{Q}(\hat{x}, t)}{\partial \hat{x}} & =0 \quad \forall \hat{x} \in \widehat{\gamma}_{b}, t>0 \\
\hat{b}_{z}(\hat{x}, 0) & =\hat{b}_{z, 0}(\hat{x})
\end{aligned}
$$

Using (10), the sediment law $\hat{Q}$ depends on the fluid velocity and can be written in the reference domain by:

$$
\begin{aligned}
\hat{Q}(\hat{x}, t) & =Q(\hat{x}+\hat{b}(\hat{x}), t) \quad \forall \hat{x} \in \widehat{\gamma}_{b} \\
& =\alpha u_{\tau}(\hat{x}+\hat{b}(\hat{x}))^{3 / 2}
\end{aligned}
$$

\subsubsection{Displacement equation}

This displacement needs to be extended in the fluid domain to associate a new ALE map over the mesh. In order to do this, we use a classical harmonic extension (see [13] for more details).

$$
\begin{aligned}
-\triangle \hat{\mathbf{d}}_{\delta} & = & 0 & \text { on } \hat{\Omega}, \\
\hat{\mathbf{d}}_{\delta} & = & 0 & \text { on } \hat{\Gamma}_{s}, \\
\frac{\partial \hat{\mathbf{d}}_{\delta}}{\partial \mathbf{n}} & = & & \text { on } \hat{\Gamma}_{i n} \cup \hat{\Gamma}_{\text {out }}, \\
\hat{\mathbf{d}}_{\delta} & =\left(0, \hat{b}_{z}(\hat{x}, t)\right)^{T} & & \text { on } \hat{\Gamma}_{b} .
\end{aligned}
$$

This allows us to have a given displacement defined on $\hat{\Gamma}_{b}$, to let free the boundaries $\hat{\Gamma}_{\text {in }}$ and $\hat{\Gamma}_{\text {out }}$, and to fix the boundary $\hat{\Gamma}_{s}$. The harmonic problem spreads the displacement $\hat{\mathbf{d}}_{\delta}$ on all the domain.

\subsubsection{Equation for $w$}

We denote by $\hat{\mathbf{w}}$ the velocity of the displacement

$$
\hat{\mathbf{w}}(\widehat{\mathbf{x}}, t)=\frac{\partial \hat{\mathbf{d}}(\hat{\mathbf{x}}, t)}{\partial t} .
$$

Then, using the ALE transformation, we can compute the velocity $\mathbf{w}$ in the domain $\Omega(t)$ :

$$
\mathbf{w}(\mathbf{x}, t)=\hat{\mathbf{w}}\left(\left(\mathcal{A}^{t}\right)^{-1}(\mathbf{x}), t\right)
$$




\section{VARiational FORMUlation}

This part is devoted to the variational formulation of the problem taking the ALE description into account.

\subsection{Variational formulation of the Exner equation}

By multiplying the Exner equation and integrating over $\hat{\gamma}_{b}$, we have the following variational formulation for equations (22)-(23): Taking a test function $\phi \in H^{1}\left(\hat{\gamma}_{b}\right)$, we have:

$$
\begin{gathered}
\frac{d}{d t} \int_{\hat{\gamma}_{b}} \hat{b}_{z}(\hat{x}, t) \phi(\hat{x}) d \hat{x}+\int_{\hat{\gamma}_{b}} \frac{\partial \hat{Q}(\hat{x}, t)}{\partial \hat{x}} \phi(\hat{x}) d \hat{x}=0 \\
\frac{d}{d t} \int_{\hat{\gamma}_{b}} \hat{b}_{z}(\hat{x}, t) \phi(\hat{x}) d \hat{x}-\int_{\hat{\gamma}_{b}} \hat{Q}(\hat{x}, t) \phi^{\prime}(\hat{x}) d \hat{x}+[\hat{Q}(\hat{x}, t) \phi(\hat{x})]_{\hat{\gamma}_{b}}=0 \quad \forall \phi \in H^{1}\left(\gamma_{b}\right) .
\end{gathered}
$$

The problem becomes: find $\hat{b}_{z}$ such that for all $\phi \in H^{1}\left(\hat{\gamma}_{b}\right)$

$$
\frac{d}{d t} \int_{\hat{\gamma}_{b}} \hat{b}_{z}(\hat{x}, t) \phi(\hat{x}) d \hat{x}=\int_{\hat{\gamma}_{b}} \hat{Q}(\hat{x}, t) \phi^{\prime}(\hat{x}) d \hat{x}-[\hat{Q}(\hat{x}, t) \phi(\hat{x})]_{\hat{\gamma}_{b}}
$$

\subsection{Variational formulation of unsteady Stokes Equation}

The problem leads to find $\mathbf{u} \in \mathbf{V}$ and $p \in Q$ such that the fluid equation (19) is satisfied. Let $\mathbf{X}$ be the functional set of test functions. Notice that the sets $\mathbf{V}, \mathbf{X}$ and $W$ will be defined later. In practice, the sets $\mathbf{V}$ and $\mathbf{X}$ can be different, they depend on the boundary conditions. Multiplying (19) with a test function $\mathbf{v} \in \mathbf{X}$ and (20) with a test function $q \in Q$, and then integrating by part, we get:

$$
\begin{aligned}
\int_{\Omega} \frac{\mathcal{D} \mathbf{u}}{\mathcal{D} t} \cdot \mathbf{v}-\int_{\Omega}[(\mathbf{w} \cdot \nabla) \mathbf{u}] \cdot \mathbf{v}+\mu \int_{\Omega} \nabla \mathbf{u}: \nabla \mathbf{v}-\mu \int_{\Gamma} \frac{\partial \mathbf{u}}{\partial \mathbf{n}} \cdot \mathbf{v}-\int_{\Omega} p \operatorname{div}(\mathbf{v})+\int_{\Gamma} p \mathbf{n} \cdot \mathbf{v} & =\int_{\Omega} \mathbf{F} \cdot \mathbf{v} \\
\int_{\Omega} \operatorname{div}(\mathbf{u}) q & =0
\end{aligned}
$$

Then, using the Reynolds transport formula on the first term of (35):

$$
\begin{aligned}
\frac{\mathrm{d}}{\mathrm{d} t} \int_{\Omega} \mathbf{u} \cdot \mathbf{v} & -\int_{\Omega}(\nabla \cdot \mathbf{w}) \mathbf{u} \cdot \mathbf{v}-\int_{\Omega}[(\mathbf{w} \cdot \nabla) \mathbf{u}] \cdot \mathbf{v} \\
& +\mu \int_{\Omega} \nabla \mathbf{u}: \nabla \mathbf{v}-\mu \int_{\Gamma} \frac{\partial \mathbf{u}}{\partial \mathbf{n}} \cdot \mathbf{v} \\
& -\int_{\Omega} p \operatorname{div}(\mathbf{v})+\int_{\Gamma} p \mathbf{n} \cdot \mathbf{v} \quad=\int_{\Omega} \mathbf{F} \cdot \mathbf{v} \\
\int_{\Omega} \operatorname{div}(\mathbf{u}) q & =0
\end{aligned}
$$


We define the following forms:

$$
\begin{array}{rlrl}
a_{1}(\mathbf{u}, \mathbf{v}) & = & \int_{\Omega(t)} \mu \nabla \mathbf{u}: \nabla \mathbf{v} d x, & \\
a_{2}(\mathbf{u}, \mathbf{v})= & -\int_{\Omega}(\nabla \cdot \mathbf{w}) \mathbf{u} \cdot \mathbf{v}-\int_{\Omega}[(\mathbf{w} \cdot \nabla) \mathbf{u}] \cdot \mathbf{v} \in \mathbf{X}, \\
a(\mathbf{u}, \mathbf{v})= & a_{1}(\mathbf{u}, \mathbf{v})+a_{2}(\mathbf{u}, \mathbf{v}) & \forall \mathbf{u} \in \mathbf{V}, \mathbf{v} \in \mathbf{X}, \\
b(\mathbf{u}, q)= & \int_{\Omega(t)} q \operatorname{div}(\mathbf{u}) d x, & \forall \mathbf{u} \in \mathbf{V}, q \in Q, \\
L(\mathbf{v})= & \int_{\Omega(t)} F(t) \cdot \mathbf{v} d x, & & \forall \mathbf{v} \in \mathbf{X} .
\end{array}
$$

We now need to treat the boundary conditions.

$$
\begin{aligned}
\mathbf{u} & =\mathbf{g}_{1} \text { on } \Gamma_{s} \\
\boldsymbol{\sigma} \mathbf{n}=\mu \frac{\partial \mathbf{u}}{\partial \mathbf{n}}-p \mathbf{n} & =\mathbf{g}_{2} \text { on } \Gamma_{\text {in }}(t) \cup \Gamma_{\text {out }}(t), \\
\mathbf{u} \cdot \mathbf{n} & =0 \quad \text { on } \Gamma_{b}(t) \\
\boldsymbol{\sigma n} \cdot \boldsymbol{\tau}=\mu \frac{\partial \mathbf{u}}{\partial \mathbf{n}} \cdot \boldsymbol{\tau} & =g_{3} \text { on } \Gamma_{b}(t) .
\end{aligned}
$$

\subsubsection{Dirichlet and Neumann boundary conditions}

We impose the Dirichlet condition in a strong way, the condition is embedded directly into the space in which we search the solution. We introduce the spaces:

$$
\begin{gathered}
\mathbf{V}=\left\{\mathbf{u} \in\left(\mathbf{H}^{1}(\Omega(t))\right)^{2}, \quad \mathbf{u}=\mathbf{g}_{1} \text { on } \Gamma_{s}\right\} \\
\boldsymbol{X}=\left\{\mathbf{v} \in\left(\mathbf{H}^{1}(\Omega(t))\right)^{2}, \quad \mathbf{v}=0 \text { on } \Gamma_{s}\right\} \\
Q=L^{2}(\Omega(t))
\end{gathered}
$$

The Neumann condition on $\Gamma_{\text {in }} \cup \Gamma_{\text {out }}$ comes naturally into the formulation. Indeed, the boundary terms can be written with $\mathbf{v} \in \boldsymbol{X}$ :

$$
\int_{\Gamma_{s}}\left(p \mathbf{n}-\mu \frac{\partial \mathbf{u}}{\partial \mathbf{n}}\right) \cdot \underbrace{\mathbf{v}}_{0}+\int_{\Gamma_{\text {in }} \cup \Gamma_{\text {out }}} \underbrace{\left(p \mathbf{n}-\mu \frac{\partial \mathbf{u}}{\partial \mathbf{n}}\right)}_{\mathbf{g}_{2}} \cdot \mathbf{v}+\int_{\Gamma_{b}} \underbrace{\left(p \mathbf{n}-\mu \frac{\partial \mathbf{u}}{\partial \mathbf{n}}\right)}_{\boldsymbol{\sigma} \mathbf{n}} \cdot \mathbf{v} .
$$

Then, the problem writes:

Find $\mathbf{u} \in \mathbf{V}, p \in Q$ such that

$$
\begin{aligned}
\frac{\mathrm{d}}{\mathrm{d} t} \int_{\Omega} \mathbf{u} \cdot \mathbf{v} & -\int_{\Omega}[(\mathbf{w} \cdot \nabla) \mathbf{u}] \cdot \mathbf{v}-\int_{\Omega}(\nabla \cdot \mathbf{w}) \mathbf{u} \cdot \mathbf{v}+\mu \int_{\Omega} \nabla \mathbf{u}: \nabla \mathbf{v}-\int_{\Omega} p \operatorname{div}(\mathbf{v}) \\
& =\int_{\Omega} \mathbf{F} \cdot \mathbf{v}+\int_{\Gamma_{\text {in }} \cup \Gamma_{\text {out }}} \mathbf{g}_{2} \cdot \mathbf{v}+\int_{\Gamma_{b}} \boldsymbol{\sigma} \mathbf{n} \cdot \mathbf{v} \quad \forall \mathbf{v} \in \boldsymbol{X}, \\
\int_{\Omega} \operatorname{div}(\mathbf{u}) q & =0 \quad \forall q \in Q .
\end{aligned}
$$


With the notations (39)- (42), the problem writes:

Find $\mathbf{u} \in \mathbf{V}, p \in Q$ such that :

$$
\begin{aligned}
\frac{\mathrm{d}}{\mathrm{d} t} \int_{\Omega} \mathbf{u} \cdot \mathbf{v}+a(\mathbf{u}, \mathbf{v})+b(\mathbf{v}, q) & =L(\mathbf{v}) \quad \forall \mathbf{v} \in \mathbf{X}, \\
b(\mathbf{u}, q) & =0 \quad \forall q \in Q .
\end{aligned}
$$

The bilinear forms $a$ and $b$ are defined by (41)-(42), and $L$ is defined by:

$$
L(\mathbf{v})=\int_{\Omega(t)} \mathbf{F} \cdot \mathbf{v}+\int_{\Gamma_{\text {in }} \cup \Gamma_{\text {out }}} \mathbf{g}_{2} \cdot \mathbf{v}+\int_{\Gamma_{b}} \boldsymbol{\sigma} \mathbf{n} \cdot \mathbf{v}
$$

\subsubsection{Slip boundary conditions}

In this section, we are interested in the interface between the fluid and the topography and we preconise to have a slip boundary condition on $\Gamma_{b}$, which is physically consistent with the sediment transport model chosen in this study, namely the bedload transport. Then, we give the variational formulation with slip boundary condition $\mathbf{u} \cdot \mathbf{n}=0$ on $\Gamma_{b}$. It is not natural to impose a slip boundary condition in the Stokes problem, and this problem has been widely studied:

\section{- First variational strategy}

A first strategy, studied in [15], consists in giving a condition on the stress tensor $\boldsymbol{\sigma n} \cdot \boldsymbol{\tau}=g_{3}$. We rewrite the test function $\mathbf{v}=(\mathbf{v} \cdot \mathbf{n}) \mathbf{n}+(\mathbf{v} \cdot \boldsymbol{\tau}) \boldsymbol{\tau}$ where $\mathbf{n}$ is the normal component and $\boldsymbol{\tau}$ is the tangential component on $\Gamma_{b}$. Taking $g_{3}=0$ and

$$
\begin{gathered}
\mathbf{u} \in \mathbf{W}=\left\{\mathbf{v} \in \mathbf{V},\left.\quad \mathbf{v} \cdot \mathbf{n}\right|_{\Gamma_{b}}=0\right\} \\
\mathbf{Y}=\left\{\mathbf{v} \in \mathbf{X},\left.\quad \mathbf{v} \cdot \mathbf{n}\right|_{\Gamma_{b}}=0\right\}
\end{gathered}
$$

it is straightforward to verify that the variational formulation writes

$$
\begin{aligned}
\frac{\mathrm{d}}{\mathrm{d} t} \int_{\Omega} \mathbf{u} \cdot \mathbf{v}-\int_{\Omega}(\nabla \cdot \mathbf{w}) \mathbf{u} \cdot \mathbf{v}-\int_{\Omega}[(\mathbf{w} \cdot \nabla) \mathbf{u}] \cdot \mathbf{v} & \\
+\mu \int_{\Omega} \nabla \mathbf{u}: \nabla \mathbf{v}-\int_{\Omega} p \operatorname{div}(\mathbf{v}) & =\int_{\Omega} \mathbf{F} \cdot \mathbf{v}+\int_{\Gamma_{\text {in }} \cup \Gamma \text { out }} \mathbf{g}_{2} \cdot \mathbf{v} \quad \forall \mathbf{v} \in \mathbf{Y}, \\
\int_{\Omega} \operatorname{div}(\mathbf{u}) q & =0 \quad \forall q \in Q .
\end{aligned}
$$

\section{- Second variational strategy}

A second strategy consists in giving a condition on the velocity at the boundary, see [28], as follows $\mu \frac{\partial \mathbf{u}}{\partial \mathbf{n}} \cdot \boldsymbol{\tau}+\alpha(\mathbf{u} \cdot \boldsymbol{\tau})=g$ with $\alpha>0$. To this aim, we notice that

$$
\int_{\Gamma_{b}} \boldsymbol{\sigma n} \cdot \mathbf{v}=\int_{\Gamma_{b}}(\boldsymbol{\sigma} \mathbf{n} \cdot \mathbf{n})(\mathbf{v} \cdot \mathbf{n})+(\boldsymbol{\sigma} \mathbf{n} \cdot \boldsymbol{\tau})(\mathbf{v} \cdot \boldsymbol{\tau})
$$

and we rewrite the test function $\mathbf{v}$ in terms of the normal component and the tangential component, as for the previous case. Taking

$$
\mathbf{u} \in \mathbf{W}=\left\{\mathbf{v} \in \mathbf{V}, \quad \mathbf{v}=0 \text { on } \Gamma_{s},\left.\quad \mathbf{v} \cdot \mathbf{n}\right|_{\Gamma_{b}}=0\right\}
$$


the variational formulation writes:

$$
\begin{aligned}
\frac{\mathrm{d}}{\mathrm{d} t} \int_{\Omega} \mathbf{u} \cdot \mathbf{v}-\int_{\Omega}(\nabla \cdot \mathbf{w}) \mathbf{u} \cdot \mathbf{v}-\int_{\Omega}[(\mathbf{w} \cdot \nabla) \mathbf{u}] \cdot \mathbf{v} & \\
+\mu \int_{\Omega} \nabla \mathbf{u}: \nabla \mathbf{v}-\int_{\Omega} p \operatorname{div}(\mathbf{v})+\int_{\Gamma_{b}} \alpha(\mathbf{u} \cdot \boldsymbol{\tau})(\mathbf{v} \cdot \boldsymbol{\tau}) & =\int_{\Omega} \mathbf{F} \cdot \mathbf{v}+\int_{\Gamma_{b}} g(\mathbf{v} \cdot \boldsymbol{\tau}) \\
& +\int_{\Gamma_{\text {in }} \cup \text { Гout }} \mathbf{g}_{2} \cdot \mathbf{v}, \quad \forall \mathbf{v} \in \mathbf{Y}, \\
\int_{\Omega} \operatorname{div}(\mathbf{u}) q & =0 \quad \forall q \in Q .
\end{aligned}
$$

\section{- Third variational strategy}

An other alternative leads to using a penalty method. As in a previous case, we take $\boldsymbol{X}=\{\mathbf{v} \in$ $\left(\mathbf{H}^{1}(\Omega(t))\right)^{2}, \quad \mathbf{v}=0$ on $\left.\Gamma_{s}\right\}$. In order to impose the condition $\mathbf{u} \cdot \mathbf{n}=0$ for the velocity which is not natural in the variational formulation, we consider the formulation (51) and penalize the natural boundary condition:

$$
\left.\boldsymbol{\sigma} \mathbf{n}\right|_{\Gamma_{b}}=-\frac{1}{\varepsilon}(\mathbf{u} \cdot \mathbf{n}) \mathbf{n}
$$

where $\varepsilon \ll 1$. The variational formulation becomes:

Find $\mathbf{u} \in \mathbf{V}$ and $p \in Q$ such that:

$$
\begin{aligned}
\frac{\mathrm{d}}{\mathrm{d} t} \int_{\Omega} \mathbf{u} \cdot \mathbf{v}+\tilde{a}(\mathbf{u}, \mathbf{v})+b(\mathbf{v}, p) & =L(\mathbf{v}), \quad \forall \mathbf{v} \in \mathbf{X}, \\
b(\mathbf{u}, q) & =0 \quad \forall q \in Q
\end{aligned}
$$

with the bilinear form $b$ defined by (42), $L$ and $\tilde{a}$ defined by

$$
\begin{aligned}
\tilde{a}(\mathbf{u}, \mathbf{v}) & =a_{1}(\mathbf{u}, \mathbf{v})+a_{2}(\mathbf{u}, \mathbf{v})+\frac{1}{\varepsilon} \int_{\Gamma_{b}}(\mathbf{u} \cdot \mathbf{n})(\mathbf{v} \cdot \mathbf{n}) d \sigma \\
L(\mathbf{v}) & =\int_{\Omega} \mathbf{F} \cdot \mathbf{v}+\int_{\Gamma_{\text {in }} \cup \text { Гout }} \mathbf{g}_{2} \cdot \mathbf{v} .
\end{aligned}
$$

It is proved by Dione in [15] that this problem converges to the problem with slip boundary conditions when $\varepsilon$ tends to zero.

\section{NumERICAL METHOD}

\subsection{Discretization in time}

We will now discretise the time derivative with a Backward Differentiation Formula (BDF) of order 1. It corresponds to the backward Euler method. Let $\Delta t$ be the time step, $t_{0}=0$ the initial time, $t_{n}=n \Delta t$ and $x^{n}$ a field at time $t_{n}$.

We can then rewrite the variational formulation at time $t_{n+1}$ :

$$
\begin{array}{rlrl}
\int_{\Omega} \frac{\mathbf{u}^{n+1}-\mathbf{u}^{n}}{\Delta t} \cdot \boldsymbol{\varphi}+\tilde{a}\left(\mathbf{u}^{n+1}, \boldsymbol{\varphi}\right)+b\left(\boldsymbol{\varphi}, p^{n+1}\right) & =L(\boldsymbol{\varphi}), & & \forall \boldsymbol{\varphi} \in \mathbf{V}, \\
b\left(\mathbf{u}^{n+1}, q\right) & =0, & \forall q \in W,
\end{array}
$$


since $\mathbf{u}^{n}$ is known, this leads to :

$$
\begin{aligned}
\int_{\Omega} \frac{1}{\Delta t} \mathbf{u}^{n+1} \cdot \boldsymbol{\varphi}+\tilde{a}\left(\mathbf{u}^{n+1}, \boldsymbol{\varphi}\right)+b\left(\boldsymbol{\varphi}, p^{n+1}\right) & =L(\boldsymbol{\varphi})+\int_{\Omega} \frac{1}{\Delta t} \mathbf{u}^{n} \cdot \boldsymbol{\varphi}, & & \forall \boldsymbol{\varphi} \in \mathbf{V}, \\
b\left(\mathbf{u}^{n+1}, q\right) & =0, & & \forall q \in W .
\end{aligned}
$$

\subsection{Discretization in space}

We consider in this section a subdivided domain $\mathcal{T}_{h}$ and the finite dimensional spaces $\mathbf{V}_{h}$ and $W_{h}$ which are the discrete spaces of $\mathbf{V}$ and $W$. As well, we approximate $\mathbf{u}$ and $p$ by $\mathbf{u}_{h}$ and $p_{h}$. We use a Galerkin approximation taking the fields $\mathbf{u}_{h}$, and $p_{h}$ in $\mathbf{V}_{h}$ and $W_{h}$, it reads:

$$
\mathbf{u}_{h}=\sum_{i=1}^{N} \alpha_{i} \boldsymbol{\varphi}_{i}, \quad p_{h}=\sum_{i=1}^{M} \beta_{i} \psi_{i},
$$

where we denote $N=\operatorname{dim} \mathbf{V}_{h}$ and $M=\operatorname{dim} W_{h}$ and $\varphi$ (resp. $\psi$ ) is the basis function of $\mathbf{V}_{h}$ (resp. $W_{h}$ ). Then, the semi discrete problem writes:

$$
\begin{aligned}
& \int_{\Omega} \frac{1}{\Delta t} \mathbf{u}_{h}^{n+1} \cdot \boldsymbol{\varphi}_{h}+\tilde{a}\left(\mathbf{u}_{h}^{n+1}, \boldsymbol{\varphi}_{h}\right)+b\left(\boldsymbol{\varphi}_{h}, p_{h}^{n+1}\right)=L\left(\boldsymbol{\varphi}_{h}\right)+\int_{\Omega} \frac{1}{\Delta t} \mathbf{u}_{h}^{n} \cdot \boldsymbol{\varphi}_{h}, \quad \forall \boldsymbol{\varphi}_{h} \in \mathbf{V}_{h}, \\
& b\left(\mathbf{u}_{h}^{n+1}, q_{h}\right)=0, \quad \forall q_{h} \in W_{h} .
\end{aligned}
$$

We choose the following compatible spaces (see [24]):

- $\mathbf{V}_{h}=\left\{\mathbf{v} \in \mathcal{C}^{0}(\bar{\Omega})\right.$ s.t. $\mathbf{v}_{\mid K} \in \mathbb{P}_{2}^{2}$ for all $\left.K \in \mathcal{T}_{h}\right\}$,

- $W_{h}=\left\{q \in \mathcal{C}^{0}(\bar{\Omega})\right.$ s.t. $q_{\mid K} \in \mathbb{P}_{1}$ for all $\left.K \in \mathcal{T}_{h}\right\} \cap L_{0}^{2}(\Omega)$,

where $\mathcal{T}_{h}$ is the set of mesh elements and $\mathbb{P}_{k}$ is the set of polynomial function of degree $k$. The space $\mathbf{V}_{h}$ can be adjusted for specific boundary conditions.

Throughout the rest of the document, we use these functionals sets.

We denote by $U^{n}, P^{n}$ the vectors

$$
U^{n}=\left(\begin{array}{c}
\alpha_{1}^{n} \\
\vdots \\
\alpha_{N}^{n}
\end{array}\right), \quad P^{n}=\left(\begin{array}{c}
\beta_{1}^{n} \\
\vdots \\
\beta_{M}^{n}
\end{array}\right),
$$

$A$ the matrix $A_{i, j}=\tilde{a}\left(\varphi_{i}, \varphi_{j}\right)$ for $1 \leq i, j \leq N$ and $B$ the divergence matrix $B_{i, j}^{T}=b\left(\varphi_{i}, \psi_{j}\right)$ for $1 \leq i \leq N, 1 \leq$ $j \leq M$. We also note $F=\left(\gamma_{i}\right)^{T}$ where $\mathbf{f}=\sum_{i=1}^{N} \gamma_{i} \boldsymbol{\varphi}_{i}$ and $M$ the mass matrix.

The problem writes:

$$
\left(\begin{array}{cc}
A+M / \Delta t & B^{T} \\
B & 0
\end{array}\right)\left(\begin{array}{l}
U^{n+1} \\
P^{n+1}
\end{array}\right)=\left(\begin{array}{c}
F \\
0
\end{array}\right)+\left(\begin{array}{cc}
M / \Delta t & 0 \\
0 & 0
\end{array}\right)\left(\begin{array}{c}
U^{n} \\
0
\end{array}\right)
$$

\subsection{Stokes-Exner coupling}

Although the algorithm for the instationary Stokes equations described in the previous section constitutes the main part of the complete method, we give in this part the complete implemented algorithm. We denote by $N_{T}$ the final time of the discrete problem and $t^{n}=n \Delta t$. Starting from an initial topography, the first step consists in solving the Stokes equation in the domain $\Omega^{n}$ delimited by the initial bottom using the method presented before. Then, denoting the velocity of the fluid at time $t^{n}$, by $\mathbf{u}^{n}$ and the velocity of the displacement of the mesh by $\mathbf{w}^{n}$, we solve the Stokes equation for this initial state. This resolution gives the numerical solution $\mathbf{u}^{n+1}$ at time $t^{n+1}$. We denote this solver by $\operatorname{StokesSolver}\left(\Omega^{n}, \mathbf{u}^{n}, \mathbf{w}^{n}\right)$. This allows to compute the new topography $b^{n+1}$ using the Exner equation and giving the velocity $\mathbf{u}^{n+1}$ and the bottom at time $t^{n}$. We note this method by $\operatorname{ExnerSolver}\left(b^{n}, \mathbf{u}^{n+1}\right)$ which allows to compute the displacement of the mesh $\Omega^{n}$ at the 
boundary $\Gamma_{b}$, that is to say at the interface. Then, it is necessary to extend the deformation in the domain to compute the new one. To do so, the method ALESolver $\left(\Omega^{n}, \mathbf{d}_{\Gamma_{b}}^{n+1}\right)$ solves the harmonic problem from the domain at time $t^{n}$ and then, gives the deformation $d^{n+1}$ that needs to be applied. Finally, the velocity $\mathbf{w}^{n+1}$ and the mesh $\Omega^{n+1}$ is computed from the displacement. At this step, all the states are obtained for the time $t^{n+1}$.

The coupled algorithm can be summarized by the following:

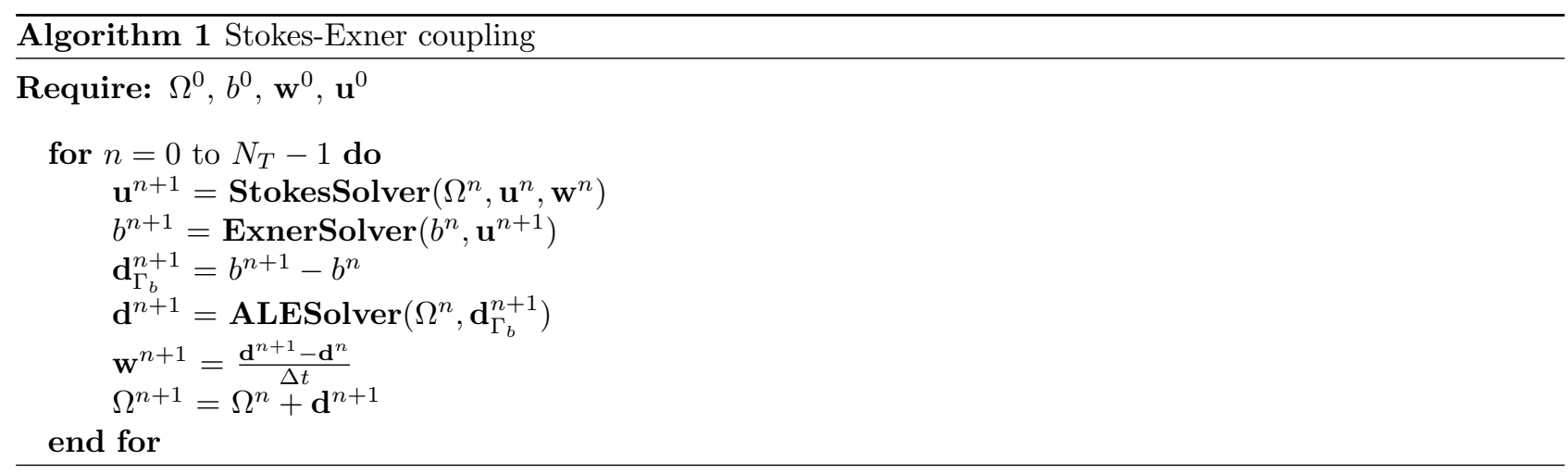

\section{Numerical TESTS}

\subsection{Validation with analytical solutions of the Stokes equations}

In order to validate the numerical method proposed and implemented with Feel++, we compare the numerical results with analytical solutions of the Stokes problem.

\subsubsection{Solution of Bercovier-Engelman}

First of all, in order to validate the implementation of the Stokes problem only, we use the solution of Bercovier-Engelman [7], which consists in finding a velocity that satisfies the free divergence condition and is null on the whole boundary. From this velocity and a source term $\mathbf{f}$, we deduce gradient pressure, and then a pressure.

$$
\begin{gathered}
\mathbf{v}=\left(\begin{array}{c}
-256 z(z-1)(2 z-1) x^{2}(x-1)^{2} \\
256 x(x-1)(2 x-1) z^{2}(z-1)^{2}
\end{array}\right) \\
p=(x-0.5)(z-0.5), \\
\mathbf{f}=\left(\begin{array}{c}
256\left(x^{2}(x-1)^{2}(12 z-6)+z(z-1)(2 z-1)\left(12 x^{2}-12 x+2\right)\right)+(z-0.5) \\
-256\left(z^{2}(z-1)^{2}(12 x-6)+x(x-1)(2 x-1)\left(12 z^{2}-12 z+2\right)\right)+(x-0.5)
\end{array}\right) .
\end{gathered}
$$

We can compare the exact solution and the approximation in Fig 3. 
$L_{2}$ errors on the velocity

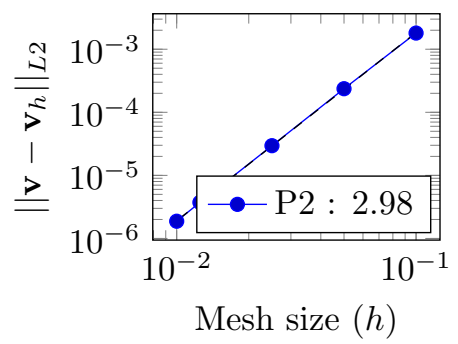

$H^{1}$ errors on the velocity

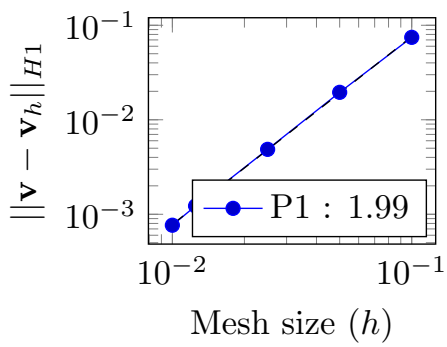

$L_{2}$ errors on the pressure

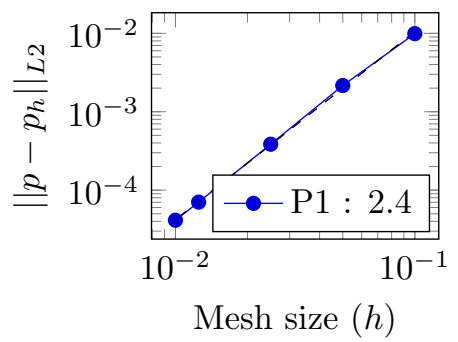

Figure 4. Convergence rates for the Bercovier-Engelman solution.

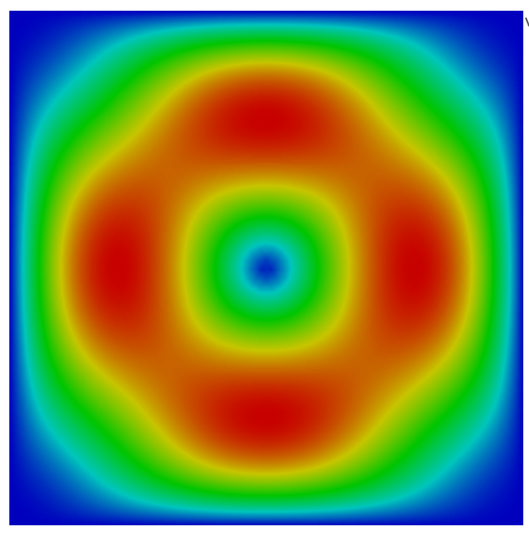

(A) Exact solution

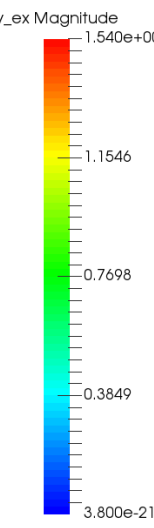

$800 \mathrm{e}-21$

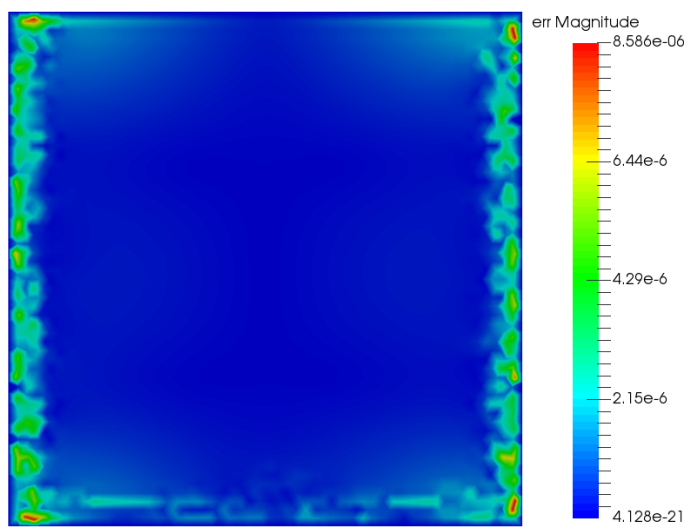

(B) Error

FIGURE 3. Velocity field of the exact solution and error with the numerical solution.

In Figure 4, we compute the errors between the exact and the computed solution and plot these errors versus the mesh size (in log-log scale). We can then verify that the method converges and that the convergence orders are 3 for the L2-norm of the velocity and 2 for the pressure, which are those expected by the theory with the finite elements chosen here.

\subsubsection{Driven cavity}

The second test case, the driven cavity, is a very classical test case in fluid dynamics. We verify again that the Stokes problem is well solved but with more physical boundary conditions that will be useful in the sequel. Indeed, to obtain this solution, we impose $\left.v\right|_{\Gamma_{i n} \cup \Gamma_{\text {out }} \cup \Gamma_{b}}=0$ and $\left.v\right|_{\Gamma_{s}}=(1,0)^{T}$. The numerical results are shown in Figure 5. 


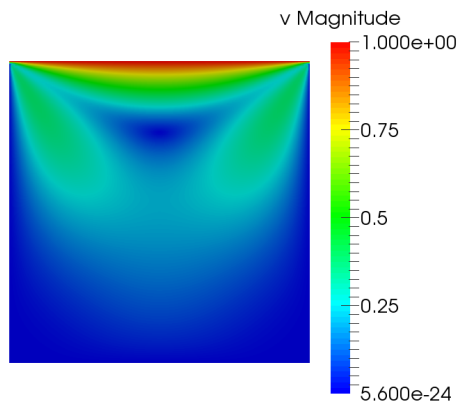

(A) Magnitude field

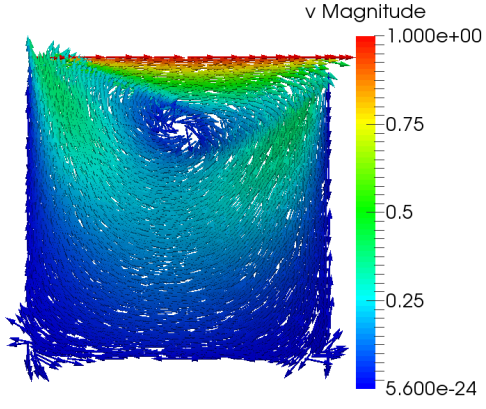

(B) Velocity field

FIGURE 5. Driven cavity.

Notice that the discontinuity of the velocity of the corners of the cavity is due to the discontinuity of the velocity imposed by the Dirichlet condition. It does not infer on the training, but to avoid this result, a polynomial function can be set instead of the constant.

\subsection{Fluvial dune test case}

To validate our complete coupled model with the Exner equation, we take an initial dune given by the equation :

$$
b_{z}(x, 0)=0.2 \times e^{-\frac{(x-2.5)^{2}}{0.2}}, \forall x \in[0, l],
$$

with $l=5$.

For the unsteady Stokes equation, we use the slip boundary condition (4)-(7) and let free the velocity at the inlet and outlet with a Neumann boundary condition (5) with $\mathbf{g}_{2}=\mathbf{0}$. On the top, we impose the same Dirichlet condition than in the driven cavity $\mathbf{u}=(1,0)^{T}$, driven the fluid to the right. For the Exner model, we use the Grass formula (10) and the initial data given by (74). We use the numerical method presented above : finite element method for spatial discretization and Implicit Euler method for time discretization for all the equations. As the cavity is driven with a moving bottom, we add the ALE formulation and obtain the results showed in Figure 6. We use the penalty method for bottom condition. The result is given in figure 6 and is similar to that of E. J. Kubatko and J. J. Westerink [31] (Fig. 2 of their paper). The same test case has been tested with multiple dunes and a similar result (distortion to the right) was obtained. This kind of solution can be difficult to represent with a numerical scheme because the solution becomes discontinuous but the algorithm stays stable during the simulation. This test case allows us to evaluate the relevance of our method but a comparison with an analytical solution is necessary to validate the method.

Remark 1. It is not straightforward to validate the method on the complete model with an analytical solution. In [31], it is explained that the Exner model is an hyperbolic equation. Indeed, we can write Exner equation (11) (with $\xi=1$ ) as:

$$
\frac{\partial b_{z}}{\partial t}+c\left(b_{z}\right) \frac{\partial b_{z}}{\partial t}=0
$$

where $c\left(b_{z}\right)=\frac{\partial Q(\mathbf{u})}{\partial b_{z}}\left(b_{z}\right)$ can be interpreted as a bed velocity (that depends on $b_{z}$ because $\mathbf{u}$ in (2)-(3) does). It is obvious that the conservative law (75) is nonlinear. It is well known that a nonlinear conservative law is sometimes subject to discontinuities. Even if the initial topography $b_{0}$ is smooth, if characteristics intersect at time $t_{d}>0$, there is no unique solution and a discontinuity can appear. For a time greater than $t_{d}$, the model no longer holds, there are several unphysical solutions. To solve this problem, it is classic to add an 


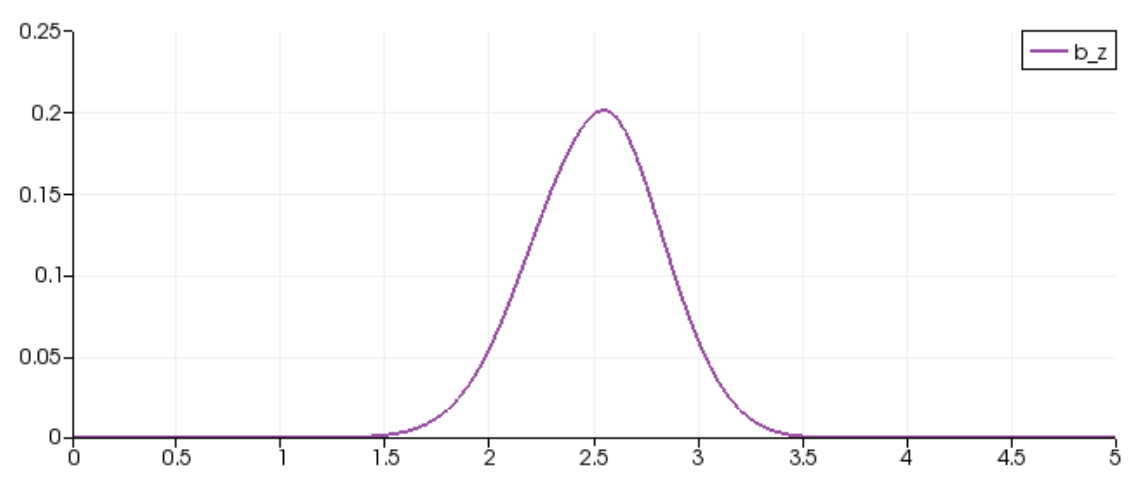

(A) $t=0 \mathrm{~s}$

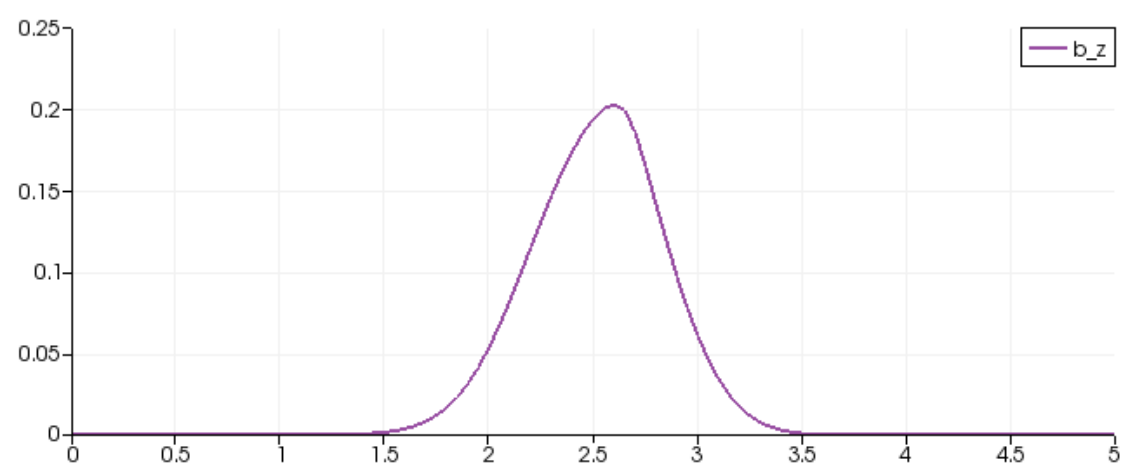

(в) $t=8 \mathrm{~s}$

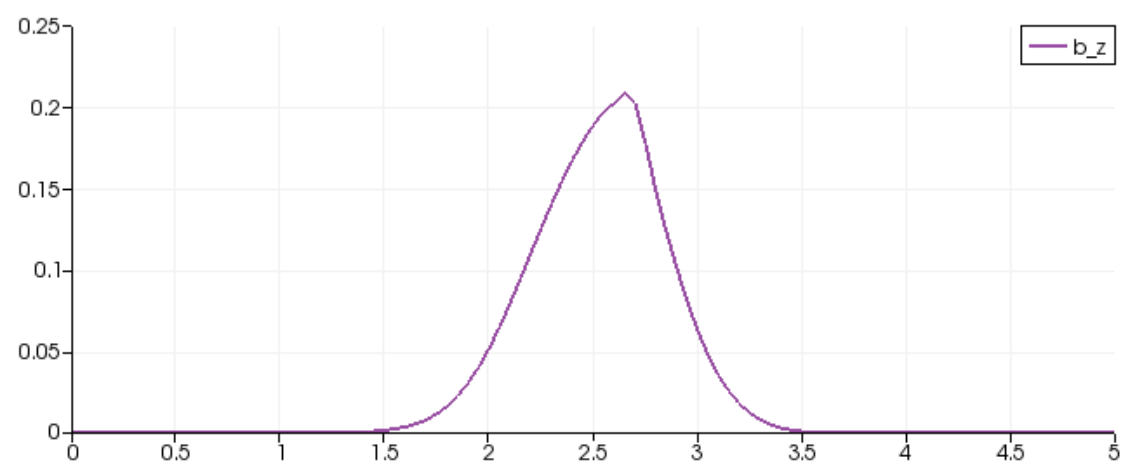

(c) $t=16 \mathrm{~s}$

FiguRE 6. Bottom topography at different times

artificial viscosity or use the integral form of the Exner model (see [31] for details). In order to avoid that kind of difficulty, we consider small simulation times and let these considerations to further studies. 


\section{Conclusion}

In this note, we consider a coupling between the Exner equation and the Stokes equations to model the transport sediments in flow phenomena. We focus on a model without free surface and use some numerical tests to evaluate the relevance of the method. The fluid structure interaction theory and method have been applied and the objective is to test the proposed method which can be extend to a free surface model. The library Feel++ and the high computing performance embedded have been used to test the solution method. Therefore, the final goal of this project is to understand the impact of the sediment transport on the flow using Navier-Stokes with a free surface system coupled with the standard Exner equation.

\section{REFERENCES}

[1] E Audusse. A multilayer Saint-Venant model: Derivation and numerical validation. Discret. Contin. Dyn. Syst. Ser. B, $5(2): 189-214,2005$.

[2] Emmanuel Audusse, François Bouchut, Marie-Odile Bristeau, and Jacques Sainte-Marie. Kinetic Entropy Inequality and Hydrostatic Reconstruction Scheme for the Saint-Venant. eprint arXiv:1409.3825, pages 1-21, 2000.

[3] Emmanuel Audusse, Christophe Chalons, Olivier Delestre, Nicole Goutal, Magali Jodeau, Jacques Sainte-Marie, Jan Giesselmann, and Georges Sadaka. Sediment transport modelling : relaxation schemes for Saint-Venant - Exner and three layer models. ESAIM: Proceedings, 38:78-98, 2012.

[4] Emmanuel Audusse, Christophe Chalons, and Philippe Ung. A simple three-wave Approximate Riemann Solver for the SaintVenant-Exner equations. working paper or preprint, September 2015.

[5] M. Larson B. Camenen. A general formula for non-cohesive bed load sediment transport. Estuarine Coastal Shelf Sci, pages 249-260, 2005.

[6] T. Belytschko and J. M. Kennedy. Computer models for subassembly simulation. Nuclear Engineering and Design, $49,1978$.

[7] Michel Bercovier and Michael Engelman. A finite element for the numerical solution of viscous incompressible flows. Journal of Computational Physics, 30(2):181-201, 1979.

[8] P. Bonneton, F. Chazel, D. Lannes, F. Marche, and M. Tissier. A splitting approach for the fully nonlinear and weakly dispersive green-naghdi model. Journal of Computational Physics, 230(4):1479 - 1498, 2011.

[9] François Bouchut. Nonlinear stability of finite volume methods for hyperbolic conservation laws and well-balanced schemes for sources. Frontiers in mathematics. Birkhäuser, Basel, Boston, Berlin, 2004.

[10] Marie-Odile Bristeau, Nicole Goutal, and Jacques Sainte-Marie. Numerical simulations of a non-hydrostatic shallow water model. Comput. Fluids, 47(1):51-64, 2011.

[11] Marie-Odile Bristeau, Anne Mangeney, Jacques Sainte-Marie, and Nicolas Seguin. An energy-consistent depth-averaged Euler system: Derivation and properties. Discret. Contin. Dyn. Syst. - Ser. B, 20(4):961-988, 2015.

[12] M. J Castro Diaz, E. D Fernandez, C Pares, and a. M Ferreiro. Two-dimensional sediment transport models in shallow water equations. A second order finite volume approach on unstructured meshes. Computer Methods in Applied Mechanics and Engineering, 198:2520-2538, 2009.

[13] Vincent Chabannes. Vers la simulation des écoulements sanguins. PhD thesis, Université de Grenoble, 2013.

[14] S. Cordier, M.H. Le, and T. Morales de Luna. Bedload transport in shallow water models: Why splitting (may) fail, how hyperbolicity (can) help. Adv. Water Resour., 34(8):980-989, 2011.

[15] Ibrahima Dione. Analyse théorique et numérique des conditions de glissement pour les fluides et les solides par la méthode de pénalisation. PhD thesis, Université de Laval, 2013.

[16] J. Donéa. Analysis of the levelling process based upon an analytic forming model. Advances Science Publishers, $3,1978$.

[17] J. Donéa, S. Giuliani, and J.-P. Halleux. An arbitrary lagrangian-eulerian fem for transient dynamic fluid-structure interactions. Computer Methods in Applied Mechanics and Engineering, 33, 1982.

[18] A. Duran and F. Marche. Discontinuous-galerkin discretization of a new class of green-naghdi equations. Communications in Computational Physics, page pp.130, 2014.

[19] R. Mueller E. Meyer-Peter. Formulae for bed-load transport. Report on the 2nd Meeting International Association Hydraulic Structure Research, pages 39-64, 1948.

[20] H.A. Einstein. The bed-load function for sediment transportation in open channel flows. Tech. Rep. 1026,US Department of Agriculture, Technical Bulletin, 1950.

[21] E. D. Fernández-Nieto, T. Morales de Luna, G. Narbona-Reina, and J. D. Zabsonré. Formal deduction of the Saint-VenantExner model including arbitrarily sloping sediment beds and associated energy. ArXiv, pages 1-44, 2015.

[22] R. M. Frank and R. B. Lazarus. Mixed eulerian-lagrangian method. Academic Press, 3, 1964.

[23] J.-F. Gerbeau and Benoit Perthame. Derivation of viscous Saint-Venant system for laminar shallow water; Numerical validation. Discret. Contin. Dyn. Syst. - Ser. B, 1(1):89-102, 2001.

[24] V. Girault and P-A. Raviart. Finite element methods for Navier-Stokes equations, volume 5 of Springer Series in Computational Mathematics. Springer-Verlag, Berlin, 1986. Theory and algorithms. 
[25] Edwige Godlewski and Pierre-Arnaud Raviart. Numerical approximation of hyperbolic systems of conservation laws. Applied mathematical sciences. Springer, New York, 1996.

[26] C Grandmont. Analyse mathématique et numérique de problèmes d'interaction fluide-structure. Application à la modélisation de l'appareil respiratoire. Habilitation à diriger des recherches, Université Pierre et Marie Curie - Paris VI, November 2009. Dans le cadre de l'ANR M3RS (ANR-08-JCJC-0013-01).

[27] J. Grass. Sediments transport by waves and currents. SERC London Cent. Mar. Technol., Report No. FL29, 1981.

[28] J. L. Guermond and P. D. Minev. A new class of massively parallel direction splitting for the incompressible Navier-Stokes equations. Computer Methods in Applied Mechanics and Engineering, 200(23-24):2083-2093, 2011.

[29] Putu Harry Gunawan and Xavier Lhébrard. Hydrostatic relaxation scheme for the 1D shallow water - Exner equations in bedload transport. Comput. Fluids, 121:44-50, 2015.

[30] A. Kalinske. Movement of sediment as bed load in rivers. Earth and Space Sciences News, pages 615-620, 1947.

[31] Ethan J. Kubatko and Joannes J. Westerink. Exact Discontinuous Solutions of Exner's Bed Evolution Model : Simple Theory for Sediment Bores. Journal of Hydraulic Engineering, 2007.

[32] D Lannes and F Marche. A new class of fully nonlinear and weakly dispersive Green-Ngaghdi models for efficient $2 \mathrm{~d}$ simulations. J. Comput. Phys., 282:238-268, 2014.

[33] P. Nielsen. Movement of sediment as bed load in rivers. Cambridge University Press, pages 615-620, 1947.

[34] P. Nielsen. Coastal Bottom Boundary Layers and Sediment Transport. Advanced Series on Ocean Engineering, vol. 4, 1992.

[35] P. Nielsen. Erosion and Sedimentation. Cambridge University Press, 1998.

[36] W. F. Noh. CEL : a time-dependent two-space-dimensional. Coupled Eulerian-Lagrangian code. Academic Press, 3, 1964.

[37] Gary Parker, Chris Paola, and Suzanne Leclair. Probabilistic Exner Sediment Continuity Equation for Mixtures with No Active Layer, 2000.

[38] B. Perthame and C. Simeoni. A kinetic scheme for the Saint-Venant system with a source term. Calcolo, 38(4):201-231, 2001.

[39] Christophe Prud'Homme, Vincent Chabannes, Vincent Doyeux, Mourad Ismail, Abdoulaye Samake, and Gonçalo Pena. Feel++: A Computational Framework for Galerkin Methods and Advanced Numerical Methods. December 2012.

[40] Christophe Prud'Homme, Vincent Chabannes, Vincent Doyeux, Mourad Ismail, Abdoulaye Samake, and Gonçalo Pena. Feel++: A computational framework for galerkin methods and advanced numerical methods. In ESAIM: Proceedings, volume 38, pages 429-455. EDP Sciences, 2012.

[41] Christophe Prud'homme, Vincent Chabannes, Stephane Veys, Vincent Huber, Cécile Daversin, Alexandre Ancel, Ranine Tarabay, Vincent Doyeux, Jean-Baptiste Wahl, Christophe Trophime, Abdoulaye Samake, Guillaume Dollé, and Alexandre Ancel. feelpp v0.98.0. May 2014.

[42] G. Smart. Sediment transport formula for steep channels. J. Hydraul, 3:267-276, 1984. 